\title{
Early identification of acute hypoxia based on brain NADH fluorescence and cerebral blood flow
}

\author{
Hua Shi*, ${ }^{*}$, Nannan Sun*,†, Avraham Mayevsky*,

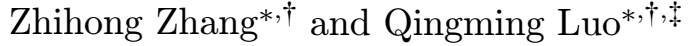 \\ *Britton Chance Center for Biomedical Photonics \\ Wuhan National Laboratory for Optoelectronics-Huazhong \\ University of Science and Technology \\ Wuhan 430074, P. R. China \\ ${ }^{\dagger}$ MoE Key Laboratory for Biomedical Photonics \\ Department of Biomedical Engineering \\ Huazhong University of Science and Technology (HUST) \\ Wuhan 430074, P. R. China \\ †qluo@mail.hust.edu.cn
}

Received 25 January 2014

Accepted 20 February 2014

Published 26 March 2014

\begin{abstract}
Hypoxia is closely related to many diseases and often leads to death. Early detection and identification of the hypoxia causes may help to promptly determine the right rescue plan and reduce the mortality. We proposed a new multiparametric monitoring method employing mitochondrial reduced nicotinamide adenine dinucleotide (NADH) fluorescence, regional reflectance, regional cerebral blood flow (CBF), electrocardiography (ECG), and respiration under six kinds of acute hypoxia in four categories to investigate a correlation between the parameter variances and the hypoxia causes. The variation patterns of the parameters were discussed, and the combination of NADH and CBF may contribute to the identification of the causes of hypoxia.
\end{abstract}

Keywords: Nicotinamide adenine dinucleotide fluorescence; acute hypoxia; early detection; cerebral blood flow.

\section{Introduction}

Hypoxia is a common pathological phenomenon, accompanying with many diseases, such as neurologic complications, ${ }^{1}$ ischemic heart disease,${ }^{2}$ acute renal failure, ${ }^{3}$ intestinal ischemia, ${ }^{4}$ pulmonary disease,${ }^{5}$ carbon monoxide poisoning, ${ }^{6}$ traumatic brain injury ${ }^{7}$ perinatal asphyxia, ${ }^{8}$ sudden cardiac death, ${ }^{9}$ etc. Severe hypoxia often leads to death. Rapid and accurate diagnosis in the early stage of hypoxia plays an important role in the treatment and prevention of severe disease development, and also determines a patient's prognosis.

This is an Open Access article published by World Scientific Publishing Company. It is distributed under the terms of the Creative Commons Attribution 3.0 (CC-BY) License. Further distribution of this work is permitted, provided the original work is properly cited. 


\section{H. Shi et al.}

Current clinically utilized finger pulse oximetry measures arterial oxygen saturation and pulse rate. ${ }^{10}$ Heart rate $(\mathrm{HR})$ variability, arterial blood gases and blood pressure ${ }^{11}$ are systemic blood oxygen indices, and unable to indicate the regional hypoxia condition in specific organs or tissue. Computed tomography (CT), Electroencephalogram (EEG) and Magnetic resonance imaging (MRI) provide evaluation of the pathological level of the whole brain. Cerebral microdialysis ${ }^{7}$ measures lactate and the lactate-pyruvate index (LPI) which are related to oxygen but the measurement is nonreal-time and time consuming. Tissue oxygen monitoring ${ }^{12}$ provides tissue oxygen tension $\left(\mathrm{P}_{\mathrm{t}} \mathrm{O}_{2}\right)$, for example, brain tissue oxygen tension, together with intracranial pressure, benefiting patient outcomes in intensive care of severe traumatic brain injury. ${ }^{13}$ However, the electrode is inserted into the brain tissue and may cause damage.

In 1962, Dr. Britton Chance pioneered a new monitoring technique which employed reduced nicotinamide adenine dinucleotide (NADH) fluorescence as a sensitive oxygen indicator in the mitochondrial level, and applied the monitoring in brain, kidney, liver, intestine and other organs in small animals. The advantages of NADH measurement are not only real time and minimally invasive, but reflects the oxygen metabolism in mitochondrial level, where the partial oxygen tension is below $1 \mathrm{mmHg}$, and much lower than $23 \mathrm{mmHg}$ in tissue level. ${ }^{14,15}$ We have provided experimental evidence that NADH fluorescence can respond to hypoxia several seconds earlier than regional reflectance, cerebral blood flow (CBF), electrocardiography (ECG) and respiration do in most hypoxia cases, depending on the way to induce hypoxia. ${ }^{16}$ Moreover, $\mathrm{NADH}$ can alarm death crisis earlier than other parameters do, ranging from several seconds to over $10 \mathrm{~min}$. And if proper and prompt judgment for the hypoxia cause was made, and right and rapid rescue was conducted, the body could be prevented from death. Therefore, the early identification of hypoxia type is crucial in clinic.

As we learned from the textbook, ${ }^{17}$ oxygen transporting to tissue goes through four steps, (1) breathed in with air, (2) combined with hemoglobin, (3) circulated with blood and (4) released from hemoglobin and utilized by tissue. Any disturbance in any step may induce hypoxia, and therefore hypoxia can be categorized into four types, called hypoxic, hypemic, circulatory and histogenous hypoxia, respectively. The monitored parameters we utilized were NADH fluorescence to represent the mitochondrial oxygenation, reflectance to indicate the blood oxygenation and cerebral blood volume (CBV), $\mathrm{CBF}$ to present the regional microcirculation, ECG to demonstrate the systemic circulation and respiration to reflect the systemic pulmonary ventilation. This paper is to find corresponding relationship between acute hypoxia causes and the monitored parameters, and suggest an early identification of acute hypoxia causes.

\section{Materials and Methods}

\subsection{Experimental protocols}

The protocol was approved by the Institutional Animal Care and Use Committee of the Huazhong University of Science and Technology. Wistar male rats, weighing $230 \pm 20 \mathrm{~g}$, were divided into 6 groups, with 8 rats in each. Anesthesia was performed by intraperitoneal injection of $10 \%$ urethane and $2 \%$ chloral hydrate $(0.9 \mathrm{~mL} / 100 \mathrm{~g}$ body weight), and sustained by additional injection of $1 / 6$ of the initial dosage when the rat appeared regaining consciousness.

The monitoring platform is the same as described in our previous paper. ${ }^{16}$ Briefly summarized, a direct current (DC) fluorometer/reflectometer combined with a laser Doppler perfusion monitor was utilized to monitor cerebral fluorescence in $450 \mathrm{~nm}$, reflectance in $366 \mathrm{~nm}$ and CBF. A corrected NADH signal was retrieved by subtracting detected intensity of $366 \mathrm{~nm}$ reflectance from that of $450 \mathrm{~nm}$ fluorescence, according to the routinely utilized calibration method introduced and consisted by Drs. Mayevsky and Chance for over 30 years. ${ }^{18}$ The excitation and emission light was transferred by a fiber probe which was fixed on the somatosensory cortex with a diameter of $3 \mathrm{~mm}$ avoiding big vessels $(>100 \mu \mathrm{m})$. Simultaneously, a multichannel physiological data acquisition and analysis system (RM6240, Chengdu Instrument Factory, Chengdu, China) was employed to detect the systemic parameters such as ECG and respiration. To achieve the simultaneous measurement, a control was conducted through a script run in LabView (National Instruments, Austin, Texas) to add markers on both output curves at the same time. Since the time constant of the integrated DC fluorometer/reflectometer 
and laser Doppler perfusion monitor is $3 \mathrm{~s}$, while the time constant of ECG recorder is only $0.2 \mathrm{~s}$, the recorded fluorescence, reflectance, corrected NADH and CBF signals were removed $3 \mathrm{~s}$ at the beginning of the time line, and the ECG was removed $0.2 \mathrm{~s}$, respectively.

Considering the four steps of oxygen transport to tissues, four kinds of hypoxia were produced by the disruption to each step. In brief, hypoxic hypoxia was demonstrated by giving only nitrogen instead of air to the rats to reduce the inhaled oxygen tension; hypemic hypoxia was developed by injection of sodium nitrate to destroy the oxygen carrying capacity; circulatory hypoxia was obtained by reducing the blood circulation to the brain; and histogenous hypoxia was induced by injection of drugs that can break the mitochondrial respiratory chain, in our case which is Chlorpromazine (CPZ). Since we have demonstrated four hypoxia death models in the previous paper, ${ }^{16}$ in this paper, to further analyze the relationship of monitored parameters with different causes of hypoxia, we added two acute hypoxia models in which the rats were rescued after induced acute hypoxia. Treatments to the six models were summarized in Table 1.

\subsection{Data process and statistics}

The monitored data of fluorescence, reflectance, corrected $\mathrm{NADH}, \mathrm{CBF}$, respiration and ECG were collected simultaneously through LabVIEW and processed to figures by MATLAB (Mathworks,
Natick, Massachusetts). The baseline of each curve was calculated as the mean value of $60 \mathrm{~s}$ before inducing hypoxia, at which time was defined as time point 0 . The baselines of fluorescence, reflectance, corrected NADH and CBF were set to $100 \%$. Empirical approach was used in order to calibrate the signal in relative units. The reflectance and fluorescence signals obtained from the photomultipliers (RCA 931B) were calibrated to a standard signal $(0.5 \mathrm{~V})$, as described in detail, ${ }^{18,19}$ by variation of photomultiplier dynode voltage obtained from a high-voltage power supply. The changes in the fluorescence and reflectance signals were calculated relative to the calibrated signals under normoxic conditions. This type of calibration is not absolute, but provides reliable and reproducible results from various animals and also among various laboratories using this approach.

The percentages of change of fluorescence, reflectance and corrected NADH were determined by the ratio of recorded voltages to $0.5 \mathrm{~V},{ }^{19}$ while the percentage of CBF change is calculated from the baseline reading. HR and respiration rate (RR) were derived from the beats during a $5 \mathrm{~s}$ interval.

After discarding the rats that failed during the experimental procedure, the numbers of rats analyzed in the paper were also shown in Table 1. IBM SPSS Statistics (IBM Corp, Armonk, New York) was utilized to perform the statistics. Results are presented as the mean \pm standard error of mean (SEM). Significance was tested by $t$-test and oneway ANOVA $(p<0.05)$.

Table 1. Summary of treatments and results of the six acute hypoxia models.

\begin{tabular}{|c|c|c|c|c|c|c|}
\hline \multirow[b]{2}{*}{ Hypoxia models } & \multicolumn{2}{|c|}{ Hypoxic } & \multirow{2}{*}{$\frac{\text { Hypaemic }}{\text { Died }}$} & \multicolumn{2}{|c|}{ Circulatory } & \multirow{2}{*}{$\frac{\text { Histogenous }}{\text { Died }}$} \\
\hline & Rescued & Died & & Rescued & Died & \\
\hline Number of rats & 8 & 8 & 6 & 7 & 5 & 7 \\
\hline Treatment & $\begin{array}{l}\text { Breathe in } \\
\mathrm{N}_{2}(30 \mathrm{~s}), \\
\text { then breathe } \\
\text { in air }\end{array}$ & Breathe in $\mathrm{N}_{2}$ & iv $100 \mathrm{mg} / \mathrm{kg} \mathrm{NaNO}_{2}$ & $\begin{array}{l}\text { Block bilateral } \\
\text { common carotid } \\
\text { arteries }(30 \mathrm{~s}), \\
\text { then release }\end{array}$ & $\begin{array}{l}\text { Cut the right } \\
\text { femoral } \\
\text { artery }\end{array}$ & $\begin{array}{l}\text { iv } 125 \mathrm{mg} / \mathrm{kg} \\
\mathrm{CPZ}\end{array}$ \\
\hline NADH Max $(\%)$ & $141.0 \pm 2.7$ & $149.3 \pm 2.1^{\mathrm{a}}$ & $126.3 \pm 2.4$ & $121.7 \pm 1.1$ & $143.0 \pm 6.1^{\mathrm{a}}$ & $144.5 \pm 3.9^{\mathrm{a}}$ \\
\hline Reflectance Min (\%) & $57.1 \pm 2.4^{\mathrm{b}}$ & $45.0 \pm 2.4$ & $59.4 \pm 2.9^{\mathrm{b}}$ & $63.4 \pm 2.5^{\mathrm{c}}$ & $69.0 \pm 8.1^{\mathrm{c}}$ & $81.9 \pm 1.8$ \\
\hline CBF Max $(\%)$ & $195.5 \pm 14.9^{\mathrm{d}}$ & $180.7 \pm 14.9^{\mathrm{d}}$ & $165.5 \pm 14.4^{\mathrm{d}}$ & & & $114.7 \pm 3.3$ \\
\hline
\end{tabular}

Notes: The CBF declined in the two circulatory models, and therefore the max values were missed.

a The NADH Max (\%) has no statistical significance between the marked three models.

b The reflectance Min (\%) has no statistical significance between the marked two models.

c The reflectance Min (\%) has no statistical significance between the marked two models.

d The CBF Max (\%) has no statistical significance between the marked three models. 


\section{Results and Discussion}

\subsection{Hypoxic hypoxia}

NADH change under acute hypoxia followed the typical time phase as reported before. ${ }^{20}$ In brief, after breathing in nitrogen, NADH rose in $3-5 \mathrm{~s}$ and reached a plateau in 15-20 s. For rats rescued by stopping nitrogen breathing and going back to normal breathing, the NADH signal dropped down to the baseline in about $30 \mathrm{~s}$, as shown in Fig. 1(a). If not, NADH curve kept at the plateau till death. It was noticed that the NADH curve declined at death time point shown in Fig. 1(b). This artificial result was due to the sudden rise of reflectance at that point, which was probably caused by cerebral cell swelling and water movement. ${ }^{19}$ Therefore, the $\mathrm{NADH}$ trend after the death time point should not be taken into account.

As stated previously, the moment when breathing ceased is named as anoxia. ${ }^{21}$ And at anoxia and death level, NADH is theoretically at the maximum level. ${ }^{22}$ However, our results showed that the maximum of NADH increase was $(141.0 \pm 2.7) \%$ in the rescued group and $(149.3 \pm 2.1) \%$ in the died group, with statistical significance $(p<0.05)$. To explain this difference, there are only two possibilities: (1) the tissue is not really anoxic at the moment ceasing breathing oxygen; or (2) NADH increases not to the maximum under anoxia. Since there is no distinction principle in clinic for anoxia and hypoxia, and our results are only based on limited number of rats, further investigation is expected.

The minimum percentage of reflectance was $(57.1 \pm 2.4) \%$ in the rescued group, and $(45.0 \pm 2.4) \%$ in the died group, also with statistical significance $(p<0.05)$. The reflectance is inverse to the absorbance of the excitation and emission light, thus depends on the amount of hemoglobin and the transition of different types of hemoglobin which change the absorbance coefficient at the detected wavelength. ${ }^{19}$ Due to the same cause of hypoxic hypoxia, the hemoglobin transition is almost the same, e.g., almost all the oxyhemoglobin transit to deoxyhemoglobin. Therefore, the significant difference was probably due to the entire employment of vessels dilution and capillary recruitment in the autoregulation of cerebral microcirculation, and indicated the more increase of CBV in died group than in rescued group.

The maximum $\mathrm{CBF}$ was $(195.5 \pm 14.9) \%$ in the rescued group and $(180.7 \pm 14.9) \%$ in the died group, respectively, but without a statistical significance $(p>0.05)$. This phenomenon suggested that the increase of CBF were similar between two groups. It was also noted that $\mathrm{CBF}$ trend was consistent with the HR at most time.

\subsection{Hypemic hypoxia}

The iv injected sodium nitrite can oxidize the normal hemoglobin to methemoglobin, which loses the

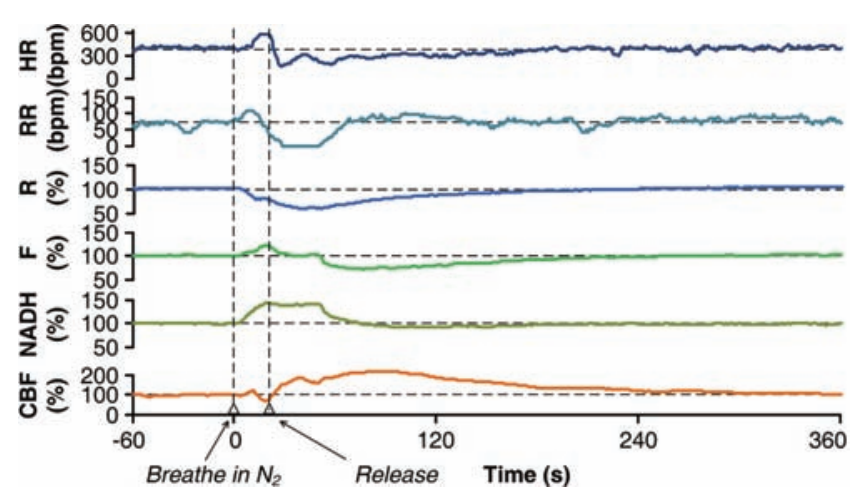

(a)

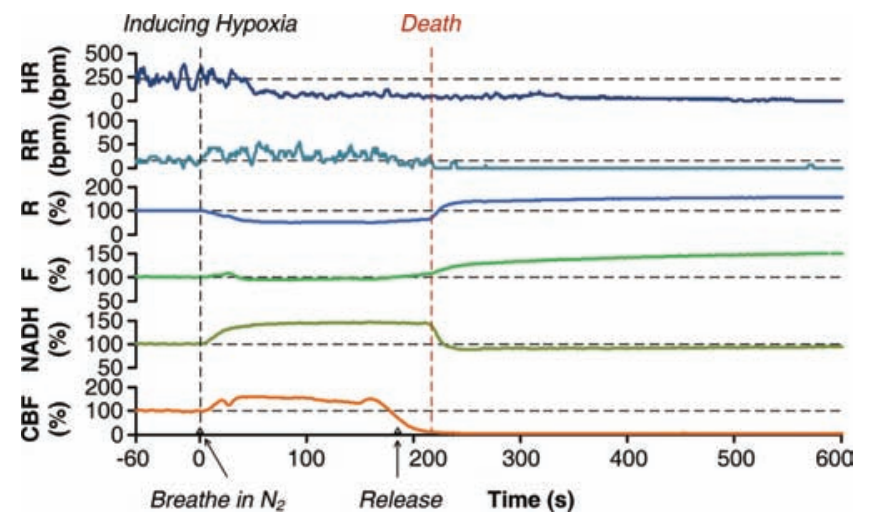

(b)

Fig. 1. Typical demonstrations of multiparametric monitoring during the procedure of hypoxic hypoxia in (a) a rescued rat and (b) a rat died in the end. The horizontal axis represents time (seconds). The rat breathed in nitrogen at the time point 0 , and the baselines, shown as horizontal dashes, was calculated as the mean values of each parameter during the $60 \mathrm{~s}$ before breathing in nitrogen. The vertical dashes tell the time when starting breathing in nitrogen (in black) and brain death (in red). Monitored parameters are listed from top to bottom as heart rate $(H R)$, respiration rate $(R R)$, reflectance change $(R)$, fluorescence change $(F)$, corrected NADH change (NADH) and cerebral blood flow change $(\mathrm{CBF})$. 
oxygen carrying capacity and reduces the oxygen transported to tissue. As shown in Fig. 2, we found that NADH signal began to rise slowly in $12-33 \mathrm{~s}$. Since methemoglobin can be reduced back to normal hemoglobin by $\mathrm{NADH}$ in the presence of NADH-methemoglobin reductase, ${ }^{23}$ when NADHmethemoglobin reductase was sufficient, NADH was actively involved in the reduction reaction of methemoglobin, representing a slight and slow rise during the induction of hypemic hypoxia and no obvious plateau was achieved. The maximum value of NADH was only $(126.3 \pm 2.4) \%$. Similarly, the $\mathrm{NADH}$ reduced after reaching its maximum was also due to the sudden increase of reflectance, which was caused by cell swelling or some other events, and therefore went out of the calibration limit and should be discarded.

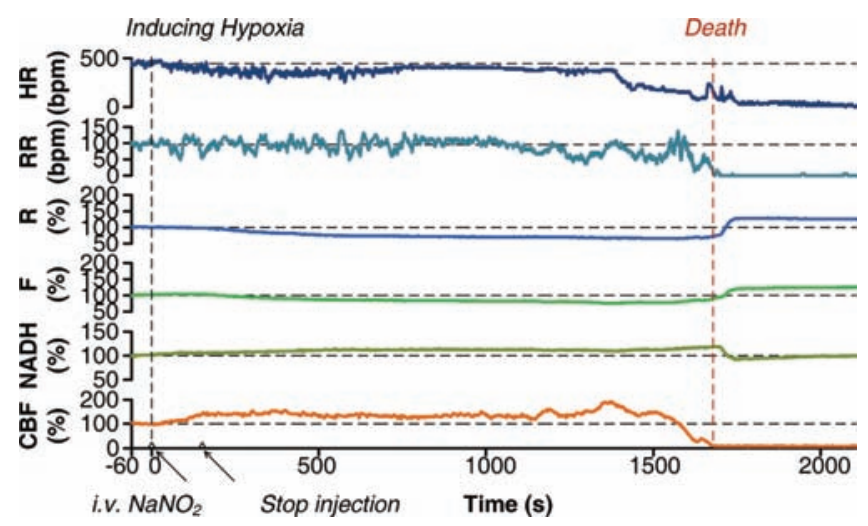

Fig. 2. Typical demonstrations of multiparametric monitoring during the procedure of hypemic hypoxia. The rat was iv injected $100 \mathrm{mg} / \mathrm{kg}$ sodium nitrate at the time point 0 .

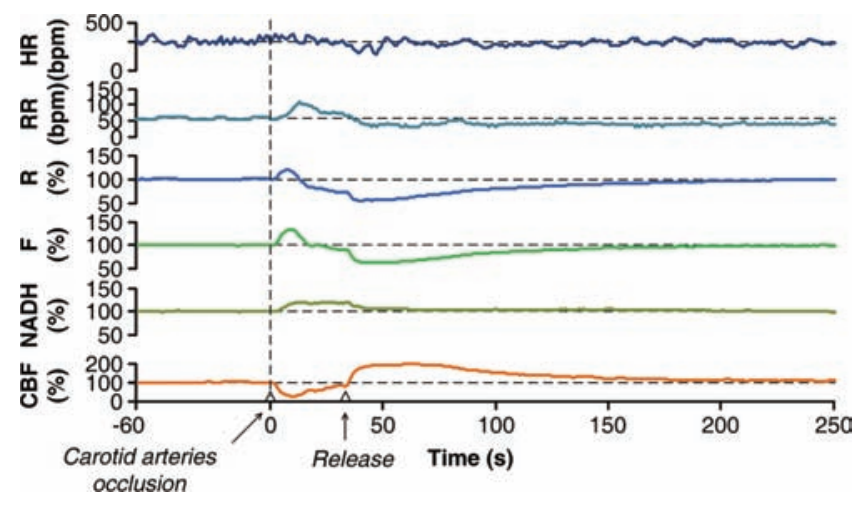

(a)
The minimum percentage of the reflectance was $(59.4 \pm 2.9) \%$. Because the molar extinction coefficient of methemoglobin is much smaller than those of either deoxyhemoglobin or oxyhemoglobin, ${ }^{24}$ the monitored reflectance reduced not that much as it did in hypoxic died group if supposing microcirculation autoregulation was similar. This inference was supported by the significance between the reflectance minimums of the two groups $(p<0.05)$.

The maximum that $\mathrm{CBF}$ reached in hypemic hypoxia group is $(165.5 \pm 14.4) \%$, but this value has no significance with $(180.7 \pm 14.9) \%$, recorded in the hypoxic died group.

\subsection{Circulatory hypoxia}

Two acute circulatory hypoxia models were established. The rescued model was reproduced by blocking the bilateral common carotid arteries for $30 \mathrm{~s}$, which prevented most of the blood supply to the brain, then releasing the arteries. While the died model was designed by cutting the right femoral artery and letting the rat bleed to death.

From Fig. 3, we can see the similarity between the period from carotid arteries occlusion and release in the rescued rat and the corresponding period in the died rat. Since there are still vertebral arteries to supply some blood to the brain, the $\mathrm{NADH}$ increase in the rescued group was only $(121.7 \pm 1.1) \%$, much lower than $(143.0 \pm 6.1) \%$, that recorded in the died group $(p<0.05)$. The latter value also showed no significant difference with it in the hypoxic died group. For similar reason, the decrease of $\mathrm{NADH}$ after reaching its maximum should be discarded. We noticed that this

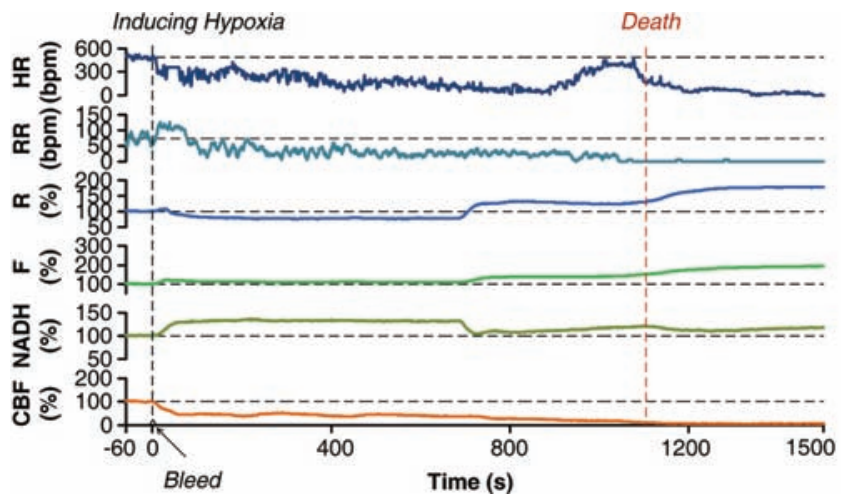

(b)

Fig. 3. Typical demonstrations of multiparametric monitoring during the procedure of circulatory hypoxia in (a) a rescued rat and (b) a rat died in the end. 
moment happened about $5 \mathrm{~min}$ before brain death, indicating that cell swelling happened much earlier than brain death. It was reasonable because cerebral edema is often induced by shock. ${ }^{25}$

Comparing the decrease percentage of reflectance in the two circulatory models, the minimums were $(63.4 \pm 2.5) \%$ and $(69.0 \pm 8.1) \%$, respectively, with no significant difference. Due to the loss of the blood, the absorbance reduced, and the reflectance rose at the beginning. Then we can see that more blood was supplied to the brain, the vital organ, controlled by the autoregulation, then reflectance appeared reduced again. The scheme presented in Fig. 3(a) is consistent with that was reported previously. ${ }^{20}$

Regarding CBF results, it was obvious in Fig. 3(a) that the CBF dropped to $(31.3 \pm 2.8) \%$, and recovered slowly due to the autoregulation. On the other hand, the group that died in the end mimicked the hemorrhagic shock procedure. As shown in Fig. 3(b), the decline of CBF contains two phases: (1) a sudden drop to $(38.1 \pm 7.1) \%$ in $68.0 \pm 7.3 \mathrm{~s}$, and (2) a slow and smooth decline to $10 \%$ (regarded as brain death $^{16}$ ) in $899.0 \pm 90.5 \mathrm{~s}$. The latter phase indicated that the autoregulation was launched to maintain the blood supply to brain tissue. We found that the $(31.3 \pm 2.8) \%$ had no significant difference with $(38.1 \pm 7.1) \%$, suggesting that the capacity of autoregulation was still sustained when CBF was reduced to about $30 \%$ of normal level.

\subsection{Histogenous hypoxia}

CPZ can easily penetrate through the blood-brain barrier, accumulate in the brain tissue and damage the respiratory chain in cerebral mitochondria. ${ }^{26}$ After a high dose iv injection, CPZ moved into cardiac and pulmonary circulation first, damaged the respiratory chain in the mitochondria of the heart and lung, representing the disorder of respiration and ECG responded before $\mathrm{CBF}$ and NADH. ${ }^{16}$ Therefore, the hypoxia to brain tissue was a mixture of circulatory and histogenous types.

$\mathrm{NADH}$ started to rise in $53.4 \pm 2.7 \mathrm{~s}$, and reached to a level of $(118.4 \pm 2.4) \%$ in $16.0 \pm 1.3 \mathrm{~s}$, then dropped back to $(109.8 \pm 1.7) \%$ in $16.3 \pm 2.1 \mathrm{~s}$, and began to rise slowly again. From Fig. 4, we can find the close correlation between NADH, CBF and $\mathrm{HR}$, suggesting that the causality existed in systemic circulation, regional cerebral circulation and regional NADH. NADH reached its maximum percentage $(144.5 \pm 3.9) \%$ before brain death, and this

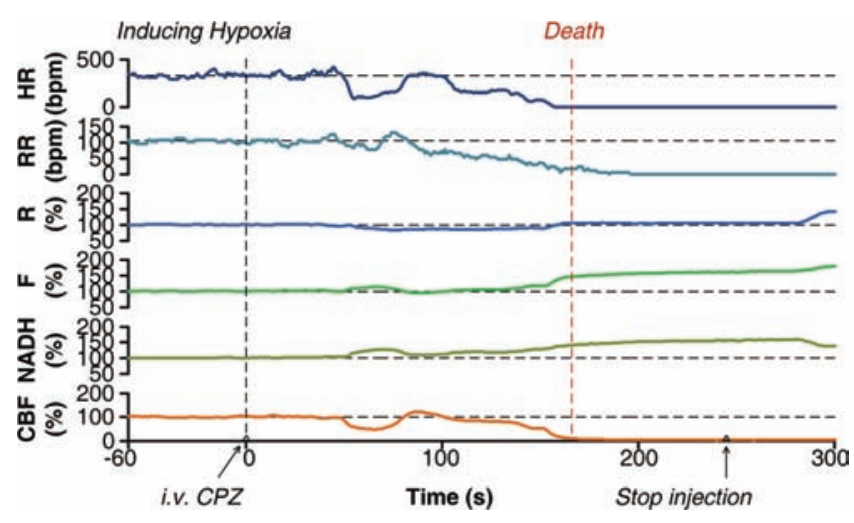

Fig. 4. Typical demonstrations of multiparametric monitoring during the procedure of histogenous hypoxia. The rat was iv injected $125 \mathrm{mg} / \mathrm{kg} \mathrm{CPZ}$ at the time point 0 .

maximum value was not significantly different from the maximum value in hypoxic died group and circulatory died group. We noticed that the artificial decrease of NADH signal appeared about 2 min after brain death, which was mainly because CPZ can prevent cerebral edema effectively. ${ }^{27}$

The changes of reflectance and $\mathrm{CBF}$ both appeared small (shown in Table 1) due to the damage of the heart and the systemic circulation.

\subsection{Correlation between monitored parameters and causes of hypoxia}

The six acute hypoxia models we presented nearly covered the majority causes of hypoxia since each of them reflected a disturbance in one of the four major steps that oxygen transported to tissue. Monitored results were presented based on single animals in Figs. 1-4, while the statistics of the maximum of $\mathrm{NADH}$, minimum of reflectance and maximum of CBF during hypoxia based on 5-8 animals were summarized in Table 1.

The maximum increase of NADH has no significant difference between the hypoxic, circulatory and histogenous died groups. Taking that NADH reaches its maximum $(100 \%$, state 5$)$ in death condition, ${ }^{22}$ we supposed the range of readings $136.9-151.4 \%$ was corresponding to that maximum $(100 \%$, state 5$)$, and therefore the normal level was limited in the range of $66.1-73.0 \%$, between state 3 and 4 . This result supported the previous inference that normal state is between state 3 and $4 .^{22}$

As we discussed, NADH correction can be interfered by cell swelling which usually accompanies 
Table 2. Temporal change of NADH, reflectance and CBF under different hypoxia conditions during the time course from inducing hypoxia to death.

\begin{tabular}{lllll}
\hline & \multicolumn{1}{c}{ Hypoxic } & Hypemic & Circulatory & Histogenous \\
\hline NADH & Prompt rise $\rightarrow$ plateau & Slow rise & Prompt rise $\rightarrow$ plateau & Slight rise $\rightarrow$ drop $\rightarrow$ slow \\
& & rise $\rightarrow$ prompt rise & Decline $\rightarrow$ rise back \\
Reflectance & Decline & Decline & Decline $\rightarrow$ rise back & Decline $\rightarrow$ rise back $\rightarrow$ slow \\
CBF & Rise $\rightarrow$ decline $\rightarrow$ rise & Slow rise $\rightarrow$ decline & Prompt decline $\rightarrow$ slow decline & decline $\rightarrow$ prompt decline \\
& $\rightarrow$ stable $\rightarrow$ decline & & & \\
\hline
\end{tabular}

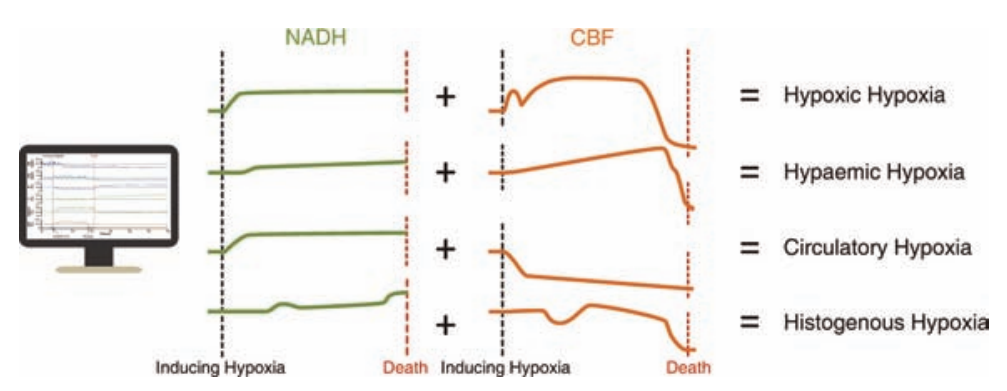

Fig. 5. The combination of the change of NADH and CBF helps in the identification of acute hypoxia causes. The change patterns of NADH and $\mathrm{CBF}$ were characterized from the four groups of animals.

with brain death. Therefore, the sudden reflectance increases should be regarded as "false" signals and the corrected NADH curve after that time point should not be taken into account. In the four death models, NADH reached the maximal level before the time at which the reflectance increase happened. The time periods ahead of the reflectance increase were $137.6 \mathrm{~s}$ in hypoxic model, $325.7 \mathrm{~s}$ in hypemic model, $596.0 \mathrm{~s}$ in circulatory model and $108.1 \mathrm{~s}$ in histogenous model, respectively. Thus, the maximum values of NADH listed in Table 1 were trustable.

The two rescued models have proved two points: (1) The change patterns of monitored parameters were similar in the same type of hypoxia, and the different cause would affect the variation. (2) If proper treatment to the specific hypoxia was conducted in time, the life can be rescued.

To establish the relationship between all monitored parameters and the causes of acute hypoxia, we summarized the temporal phases of NADH, reflectance and CBF in Table 2 for the four death models of hypoxia. For RR and HR, the patterns were complex and were excluded. The combination of NADH and CBF could provide sufficient judgment to the identification of acute hypoxia causes, as demonstrated in Fig. 5.

\section{Conclusion}

We have established six acute hypoxia models, covering each major obstruction of oxygen transport steps. By monitoring the regional mitochondrial NADH fluorescence, regional microcirculatory $\mathrm{CBF}$ and reflectance, systemic ECG and systemic respiration, we summarized the variation patterns under different acute hypoxia, and suggested a possible early identification of hypoxia cause by combining NADH and CBF indices. Since we have demonstrated that with accurate and prompt judgment of hypoxia cause and right treatment, the life can be saved, ${ }^{16}$ this identification will help to promote the clinical application of the multiparametric monitoring method.

Moreover, the measurement can be applied to other organs or tissues with an adapted fiber probe. Demonstration of some application were reported, ${ }^{28}$ but further investigation on the corresponding identification judgment should be carried on in the future.

\section{Acknowledgment}

We thank late Prof. Britton Chance for his leading and sustained contribution to the NADH fluorescence 
technique. And hope he will be happy to see NADH measurement has been inherited in Britton Chance Center for Biomedical Photonics. Prof. Avraham Mayevsky is on leave from The Mina \& Everard Goodman Faculty of Life Sciences, Bar-Ilan University, 52900 Ramat Gan, Israel, and is supported by the 111 Project of China (B07038). This work is also supported by the Ph.D. Programs Foundation of the Ministry of Education of China (Grant No. 20110142130006) and the Director Fund of Wuhan National Laboratory for Optoelectronics (WNLO, 2009, Z.H. ZHANG).

\section{References}

1. I. Erol, F. Alehan, F. Ozcay, O. Canan, M. Haberal, "Neurological complications of liver transplantation in pediatric patients: A single center experience," Pediatr. Transplant. 11(2), 152-159 (2007).

2. B. Ostadal, "The past, the present and the future of experimental research on myocardial ischemia and protection," Pharmacol. Rep. 61(1), 3-12 (2009).

3. C. M. Cheung, A. Ponnusamy, J. G. Anderton, "Management of acute renal failure in the elderly patient," Drug. Aging 25(6), 455-476 (2008).

4. P. Renner, K. Kienle, M. H. Dahlke, P. Heiss, K. Pfister, C. Stroszczynski, P. Piso, H. J. Schlitt, "Intestinal ischemia: Current treatment concepts," Langenbeck. Arch. Surg. 396(1), 3-11 (2011).

5. G. L. Semenza, "Oxygen-regulated transcription factors and their role in pulmonary disease," Resp. Res. 1(3), 159 (2000).

6. G. Oliu, S. Nogue, O. Miro, "Carbon monoxide poisoning: Pathophysiologic principles underlying good treatment," Emergencias 22(6), 451-459 (2010).

7. I. Timofeev, M. Czosnyka, K. L. Carpenter, J. Nortje, P. J. Kirkpatrick, P. G. Al-Rawi, D. K. Menon, J. D. Pickard, A. K. Gupta, P. J. Hutchinson, "Interaction between brain chemistry and physiology after traumatic brain injury: Impact of autoregulation and microdialysis catheter location," J. Neurotrauma. 28(6), 849-860 (2011).

8. E. Borg, "Perinatal asphyxia, hypoxia, ischemia and hearing loss: An overview," Scand. Audiol. 26(2), 77-91 (1997).

9. R. J. Sung, M. R. Lauer, "Sudden cardiac death syndrome: Diagnosis and management," J. Formos. Med. Assoc. 99(11), 809-822 (2000).

10. T. O'connor, G. Dubowitz, P. E. Bickler, "Pulse oximetry in the diagnosis of acute mountain sickness," High Alt. Med. Biol. 5(3), 341-348 (2004).

11. M. S. Koehle, J. A. Guenette, D. E. Warburton, "Oximetry, heart rate variability, and the diagnosis of mild-to-moderate acute mountain sickness," Eur. J. Emerg. Med. 17(2), 119-122 (2010).

12. A. B. Valadka, J. C. Goodman, S. P. Gopinath, M. Uzura, C. S. Robertson, "Comparison of brain tissue oxygen tension to microdialysis-based measures of cerebral ischemia in fatally head-injured humans," J. Neurotrauma. 15(7), 509-519 (1998).

13. M. F. Stiefel, A. Spiotta, V. H. Gracias, A. M. Garuffe, O. Guillamondegui, E. Maloney-Wilensky, S. Bloom, M. S. Grady, P. D. Leroux, "Reduced mortality rate in patients with severe traumatic brain injury treated with brain tissue oxygen monitoring," J. Neurosurg. 103(5), 805-811 (2005).

14. J. Nunn, "Cerebral oxygen transport consumption and metabolism: Transport of oxygen to the brain: Respiratory, circulatory and biochemical factors," Acta Anaesth. Scand. 15(s45), 69-77 (1971), "Transport of oxygen and carbon dioxide in blood and tissue fluids", Chap. 40, Textbook of Medical Physiology, A. C. Guyton, J. E. Hall, Eds., pp. 502513, Elsevier Health Sciences, Philadelphia (2006).

15. B. Chance, N. Oshino, T. Sugano, A. Mayevsky, "Basic principles of tissue oxygen determination from mitochondrial signals," Adv. Exp. Med. Biol. 37, 277-292 (1973).

16. H. Shi, N. Sun, A. Mayevsky, Z. Zhang, Q. Luo, "Preclinical evidence of mitochondrial nicotinamide adenine dinucleotide as an effective alarm parameter under hypoxia," J. Biomed. Opt. 19(1), 017005 (2014).

17. G. J. Tortora, B. H. Derrickson, Principles of Anatomy and Physiology, John Wiley \& Sons (2008).

18. A. Mayevsky, B. Chance, "Intracellular oxidationreduction state measured in situ by a multichannel fiber-optic surface fluorometer," Science 217(4559), 537 (1982).

19. A. Mayevsky, G. G. Rogatsky, "Mitochondrial function in vivo evaluated by NADH fluorescence: From animal models to human studies," Am. J. Physiol. Cell Physiol. 292(2), C615-C640 (2007).

20. A. Mayevsky, "Mitochondrial function and energy metabolism in cancer cells: Past overview and future perspectives," Mitochondrion 9(3), 165-179 (2009).

21. B. Chance, P. Cohen, F. Jobsis, B. Schoener, "Intracellular oxidation-reduction states in vivo," Science 137(3529), 499-508 (1962).

22. A. Mayevsky, E. Barbiro-Michaely, "Shedding light on mitochondrial function by real time monitoring of NADH fluorescence: I. Basic methodology and animal studies," J. Clin. Monit. Comput. 27(1), 1-34 (2013).

23. E. M. Scott, I. W. Duncan, V. Ekstrand, "The reduced pyridine nucleotide dehydrogenases of human erythrocytes," J. Biol. Chem. 240(1), 481485 (1965). 
24. M. F. Perutz, E. J. Heidner, J. E. Ladner, J. G. Beetlestone, C. Ho, E. F. Slade, "Influence of globin structure on the state of the heme. III. Changes in heme spectra accompanying allosteric transitions in methemoglobin and their implications for hemeheme interaction," Biochemistry 13(10), 2187-2200 (1974).

25. G. T. Trevisani, S. R. Shackford, J. Zhuang, J. D. Schmoker, "Brain edema formation after brain injury, shock, and resuscitation: Effects of venous and arterial pressure," J. Trauma Inj. Infect. Crit. Care 37(3), 452-458 (1994).
26. M. Dawkins, J. Judah, K. Rees, "The effect of chlorpromazine on the respiratory chain. Cytochrome oxidase," Biochem. J. 72(2), 204 (1959).

27. P. H. Bulle, "Effects of reserpine and chlorpromazine in prevention of cerebral edema and reversible cell damage," Exp. Biol. Medi. 94(3), 553-556 (1957).

28. A. Mayevsky, E. Barbiro-Michaely, "Shedding light on mitochondrial function by real time monitoring of NADH fluorescence: II: Human studies," J. Clin. Monit. Comput. 27(2), 125-145 (2013). 